\title{
Development of K4 Correlator for Pulsar VLBI: Japan-Russia Baseline
}

\author{
M. Sekido, S. Hama, H. Kiuchi, M. Imae, Y. Hanado, \& Y. Takahashi \\ Communications Research Laboratory, 893-1 Hirai, Kashima Ibaraki 314 Japan
}

A. E. Rodin \& Y. P. Ilyasov

Lebedev Physical Institute, Pushchino Radio Observatory, Russia

Abstract. We are doing astrometric pulsar VLBI observation with Kashima-Kalyazin 7000 $\mathrm{km}$ baseline. K4 correlator is under the development for this observation program. When XF type correlator is used for pulsar processing with gating, attention should be paid to avoid fluctuation on delay result due to fractional bit effect. This influence is serious around the point that bit shift for delay tracking and pulsar period is synchronized. In this paper, the K4 correlation system is introduced and fractional bit effect on pulsar processing is explained.

\section{Introduction}

VLBI observations of pulsars are not only a useful tool to investigate pulsar's magneto-sphere (cf. Bartel et al. 1995, Gwinn et al., these Proceedings, p. 327), but also allows us to compare the extra-galactic coordinate system defined by quasars and the coordinate system based on planetary ephemeris, since millisecond pulsars are one of the few types of objects whose positions are determined precisely in both systems (cf. Bartel et al. 1996, Dewey et al. 1996). We are doing VLBI observation of pulsars with Kashima-Kalyazin baseline. Our purpose is measurement of precise position and proper motion of pulsars, which is important for studying the relation between pulsars and their progenitor supernova remnants (Lyne \& Lorimer 1994). Difficulties of pulsar VLBI are due to the large negative spectral index and the weak flux density. Therefore pulsar gating at correlation processing is essential to get fringes from weak pulsar signals.

\section{The K4 Correlator System}

The overview of K4 correlation system is shown in Figure 1. At present this system is capable of single baseline processing. The $\mathrm{K} 4$ correlator is a XF type correlator with 18 lags for each frequency channels. It can process 16 channels, $16 \mathrm{Msps}, 1$ bit data or 8 channels, $16 \mathrm{Msps}, 2$ bit data. Then the maximum total processing rate is $256 \mathrm{Mbps}$. $64 \mathrm{Mbps}(4 \mathrm{Mbps} \times 16$ channels) mode is used in our Pulsar VLBI experiment.

\subsection{Pulsar Gate}

The correlator has gating function for millisecond pulsar data. Pulsar gate must work synchronizing with pulse arrival time for effective improvement of the correlation amplitude. Then the gating period should be accurate more than $10^{-7}$ (i.e. 7 significant digits is necessary) for millisecond pulsars. Therefore our arrival time calculation program takes into account not only earth's orbital motion but also binary pulsar's orbital motions if the target pulsar is in a binary system. 


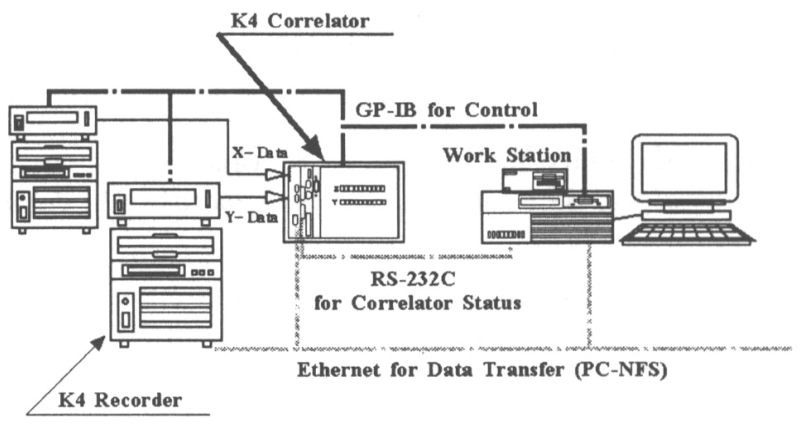

Figure 1. The K4 correlation system: The correlator is controlled by UNIX workstation via GP-IB. The correlation data are transferred from correlator to the workstation by Ethernet.

\subsection{Fractional Bit Effect}

Fractional bit compensation is inevitable and attention have to be paid to this point if correlator is XF type. In usual correlation mode, when bit shift occurs frequently in a single accumulation period (AP), fractional bit effect is averaged out. But in case of fringe rate is very small (i. e. there are almost no bit shift), the effect will not be averaged out in an AP, and then beat between AP and bit shift will appear. This effect is more serious in pulsar gating mode. Especially in case pulsar period is close to bit period (e.g., second pulsar), beat like fluctuation will disturb the fringe phase of the data. Firstly this effect was pointed out by Roger Cappallo at Haystack Observatory. To avoid this effect, we have to choose AP as long as possible to differentiate the two frequencies at the step of correlation.

\section{Conclusion}

We are developing K4 correlator with gating function for pulsar VLBI observation. Attention should be paid to the relation between bit shift period and pulsar period when XF type correlator is used for pulsar data processing.

Acknowledgments. B. A. Poperechenko, M. Dorgov, A. Fateev of Moscow Power Engineering Institute, V. V. Oreshko and other staffs of Pushchino Radio Observatory of Astro Space Center have helped us at Kalyazin $64 \mathrm{~m}$ station operation. Dr. J. Nakajima and E. Kawai of Kashima $34 \mathrm{~m}$ antenna maintenance team have been supporting our experiment. We thanks to them very much. We also appreciate to R. J. Cappallo at Haystack Observatory and T. Kondo at CRL for useful discussion about fractional bit effect on correlator.

\section{References}

Bartel, N., et al. 1995. $A J, \mathbf{9 0}, 2532-2539$.

Bartel, N., et al. 1996. $A J, 112,1690-1696$.

Dewey, R. J., et al. 1996. $A J, 111,315-319$.

Lyne, A. G., \& Lorimer, D. R. 1994. Nature, 369, 127-129. 